\title{
Temporal discrimination factors in the delayed matching-to-sample task in monkeys
}

\author{
ROBERT W. WORSHAM \\ Ramapo College, Mahwah, New Jersey 07430
}

\begin{abstract}
The temporal discrimination hypothesis (TDH) of delayed matching-to-sample (DMTS) stresses the animal's ability to discriminate which choice-stimulus alternative has appeared most recently as sample. Thus, the emphasis is placed on discriminative processes, temporal in nature, rather than on the traditional trace or buffer storage mechanisms of short-term memory. Some of the predictions of the TDH were tested within the context of the DMTS task. Experiment I showed that the difficulty of sample-stimulus sequences could be predicted by the TDH. Experiment II showed DMTS performance to be an increasing function of the number of sample stimuli employed, a result predicted by the TDH, but not by a traditional proactive interference interpretation. The results demonstrate the importance of temporal discriminative processes in DMTS. The possibility for a simpler theoretical approach to memory, in general, is discussed.
\end{abstract}

Some theorists interpret short-term memory (STM) phenomena within the framework of the traditional long-term memory (LTM) concepts of proactive and retroactive interference, thus precluding the necessity of postulating separate processes unique to STM. Others believe that separate processes are at work in STM, and that some sort of "trace" or "buffer" must be postulated in order to account for memory failure after short durations of time (e.g., Atkinson \& Wickens, 1971; Broadbent, 1958, 1963, 1971; Brown, 1958; Murdock, 1972).

With the proliferation of experiments addressing themselves to identifying and explaining processes involved in STM, the seemingly simple initial concept of a trace or buffer has had to become increasingly more complex to explain certain results. For example, Salzberg, Parks, Kroll, and Parkinson (1971) found that when a $12-\mathrm{sec}$ retention interval was filled with an auditory shadowing task, recall was far better when the target items (letters of the alphabet) were presented visually rather than verbally. Massaro and Kahn (1973) have presented evidence that recognition memory for a tone was adversely affected by presentation of another tone within the retention interval whereas presentation of a light stimulus had no such effect. Also, Worsham and D'Amato (1973) found that monkeys' performance in a delayed matching-to-sample (DMTS) task was reduced drastically when retention intervals were spent in a houselight-on condition, rather than in darkness. No such decrements were found when white noise or recorded monkey vocalizations occurred during

This research was supported by National Science Foundation Grant GB-24386X to M. R. D'Amato, whom I wish to thank for his careful guidance and reading of the manuscript, and by a National Institute of Mental Health Predoctoral Fellowship to the author. The experiments reported were part of a doctoral dissertation presented to Rutgers University, New Brunswick, New Jersey. Requests for reprints should be addressed to the author, School of Theoretical and Applied Science, Ramapo College, Mahwah, New Jersey 07430. retention intervals. These data suggest the possibility of separate STM stores for different types of stimuli, or at least for different stimulus modalities. The extreme of this type of interpretation is seen in Wickelgren's (1970) multitrace strength theory of memory, which says that every sensory, motor, and cognitive modality of performance is a modality of memory, and that different stores exist for the different modalities.

The overriding emphasis in the STM literature has been on storage processes, with a variety of functional and structural mechanisms advanced to account for the observed properties of STM. The role of temporal discrimination processes has been largely overlooked (but see Murdock, 1972). However, a great deal of what we regard as STM, both in and out of the laboratory, may actually rest on the ability to form accurate temporal judgments. In a DMTS task, for example, monkeys are required on each trial to decide which of two or more comparison stimuli has appeared most recently as the sample stimulus. If the animal can make this "recency" discrimination, then it has "remembered" what the sample stimulus for that trial had been. In human STM experiments, the subject is often required to produce the digit or letter that was presented to him most recently as the target stimulus. The fact that recognition is required in the one case and recall in the other seems to be of little importance. In both of these cases, the subject is essentially being required to form a temporal discrimination among highly discriminable items which are heavily overlearned. Thus, forgetting may occur not so much because of decay or interference per se, but because it is difficult to place each item in the proper temporal sequence of target stimuli. This interpretation of forgetting is specifically referred to as the "temporal discrimination hypothesis" (D'Amato, 1973).

Other investigators have suggested that some types of forgetting can be viewed as a failure of temporal 


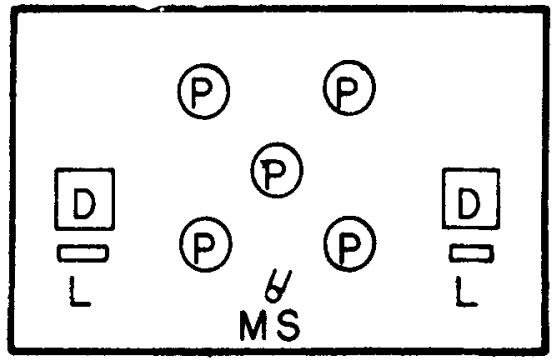

Figure 1. Schematic of the response panel in the testing chamber. The five inline projectors (P) were fitted with clear-plastic response keys. To either side of the panel were light displays (D), beneath which were response levers (L). The microswitch (MS) was a tubular piece of metal protruding from the panel, with which trials could be initiated by moving it back and forth.

discrimination. Weiskrantz (1970) has suggested the importance of recency discrimination in both the delayed-response task and DMTS, two traditional paradigms for studying STM in animals. Gleitman (1971) and Winograd (1971) have likewise discussed the role of recency discriminations in regard to LTM paradigms for animals.

The purpose of the following experiments was to explore some of the implications and predictions of the temporal discrimination approach to STM within the context of the DMTS task.

\section{EXPERIMENT I}

The ability of the animal to discriminate which of two stimuli (sample and comparison) it has seen most recently as sample should depend not only on the absolute retention interval length, but also on the sample sequence of preceding trials. For example, if the incorrect choice alternative of a given trial had not appeared as sample on any of the immediately prior trials, then the discrimination of the stimulus seen most recently as sample on the present trial should be an easy one.

In the present experiment, the importance of sample sequence was investigated by creating "easy" and "hard" sample sequences as predicted by the temporal discrimination hypothesis. A hard sample sequence should be one in which the incorrect comparison stimulus for a given trial occurred as the sample stimulus on the immediately preceding trial. An easy sample sequence should be one in which the incorrect comparison stimulus for a given trial has not appeared as a sample in the immediately preceding trials.

\section{Method}

Subjects. Six capuchin monkeys (Cebus apella) were used, three males and three females. All were well experienced with the testing situation, having been used in DMTS and visual discrimination tasks for the previous 5 years. They were maintained on Purina Monkey Chow (25\% protein) at between $90 \%$ and $100 \%$ of their full-ration body weights.

Apparatus. Testing of the subjects took place in a Lehigh Valley primate chamber, one wall of which had been fitted with inline projectors (IEE), light display panels, and manipulanda as shown in Figure 1. A foodcup for delivering 190-mg banana pellets was located on the wall to the right of the intelligence panel. The chamber houselight provided three highly discriminable levels of illumination: (1) in the houselight-on condition, a $15-\mathrm{W} 115-\mathrm{V}$ ac bulb, filtered by smoked plastic provided the brightest level; (2) in the houselight-dim condition, the illumination was reduced to one-sixth of the houselight-on condition; (3) in the houselight-off condition, the chamber was almost totally dark.

All programming of stimulus events and response contingencies was handled online by a PDP 8/e computer. Animals were monitored over closed-circuit television during testing in order to identify competing behaviors which might invalidate a test session (i.e., nonattention to the sample stimulus).

Procedure. Since most of the procedures are common to both experiments, they will be described in some detail here. The subject initiated a trial by activating a microswitch lever 15 times. For some animals, completion of the FR 15 on the microswitch resulted in the sample stimulus being presented on the center projector and remaining there until the subject pressed the plastic key superimposed over it. This terminated the sample stimulus and commenced a 2 -sec retention (delay) interval which was spent in darkness. For the other animals, completion of the FR 15 resulted in the display unit (D in Figure 1) above the left lever being illuminated. When the animal pressed the left lever, the sample stimulus was presented on the center projector for $.75 \mathrm{sec}$ followed by entry into the 2-sec darkened retention interval. For all animals, the end of the retention interval was signaled by the houselight-on condition, with the sample stimulus and one comparison stimulus being presented on two of the outer four projectors. A correct response (pressing the plastic key over the sample stimulus) was rewarded by a banana pellet, while a response to the comparison was not. Either type of response (correct or incorrect) caused termination of the two stimuli, and the subject was free to initiate the next trial at any time during this intertrial interval (ITI), which was spent with the houselight on.

Appropriate controls were employed to prevent the possibility of pseudomatching (D'Amato \& O'Neill, 1971). The two different methods of sample presentation were employed because of the animals' previous DMTS experience; Lucy, Fifi, and Coco were familiar with the former method, while Jocko, Roscoe, and Peewee were familiar with the latter. This difference was not considered a variable, as D'Amato and Worsham (1972) have shown that sample input duration does not affect DMTS performance.

The stimuli used were red disk, blue disk, triangle, vertical line, horizontal line, square, circle, dot, and plus. Animals were pretrained on DMTS with these stimuli for a minimum of 72 trials at 2-sec delays, and when formal testing began, four subjects were performing at a level of at least $96 \%$ correct, while two were performing at $92 \%$.

With pretraining completed, formal testing began; this consisted of six daily sessions of DMTS, 24 trials per session. The independent variable involved two arrangements of sample sequences. For "easy" sequences, the comparison stimulus on any given trial had not appeared as the sample stimulus for at least three trials previous, and in most cases, four. In "hard" sequences, the comparison stimulus on any given trial almost always occurred as the sample stimulus on the immediately preceding trial. These "hard" sequences would supposedly maximize confusion in discriminating the stimulus seen most recently as sample. Easy and hard sequence arrangements alternated over the six sessions, beginning with an easy session. 


\section{Results and Discussion}

The performance levels achieved with easy and hard sequences are shown in Figure 2. As predicted, performance was significantly poorer on the hard sequences, as shown by an analysis of variance, $F(1,24)=17.062, p<.01$. The main effect of subjects, which was considered a fixed effect since they were neither naive nor randomly selected, was not significant. However, the sequence-by-subjects interaction did prove to be significant, $F(5,24)=2.662, p<.05$.

These results demonstrate the effectiveness of sample-stimulus sequences as a variable in DMTS, presumably because the sequencing of sample stimuli affects the ability of the animals to form appropriate temporal discriminations. In the hard sequences, animals found it more difficult to discriminate which stimulus had been seen more recently as sample because the sample and comparison stimuli occurred relatively close together temporally in the role of sample. Guaranteeing a substantial temporal separation, the easy sequences facilitated formation of the appropriate temporal discriminations. It should be stressed that the difficulty variable emerged as highly significant, despite the extremely short duration of the retention interval $(2 \mathrm{sec})$. Previous data from our laboratory have shown that these animals usually perform close to $100 \%$ correct on 2-sec delays when stimulus sequences are randomized.

The decrement associated with the hard sequences is related to proactive interference in that an event of a previous trial interferes with performance on the current trial. The temporal discrimination hypothesis attempts to identify the mechanism behind this "interference," namely, impairment of the temporal discrimination processes upon which successful performance is based.

A secondary finding of this experiment is the ease with which the six animals generalized the concept of DMTS to new stimuli. Previous studies had limited sample-set size to four stimuli (red field, triangle, vertical line, and square). The five additional stimuli were employed in the present study simply to meet the requirement of temporal separation within the easy sample sequences. The existence of the DMTS "learning set" was evidenced by the minimal amount of pretraining necessary. Herman and Gordon (1974) have pointed out the lack of available data on DMTS learning sets; hence, this informal observation may be useful to others.

\section{EXPERIMENT II}

If there is any substance in the temporal discrimination hypothesis, DMTS performance ought to be better with larger sample sets. The reason is clear. With a small sample set, say two stimuli, it will frequently be the case that the incorrect comparison stimulus will have appeared as sample a relatively short

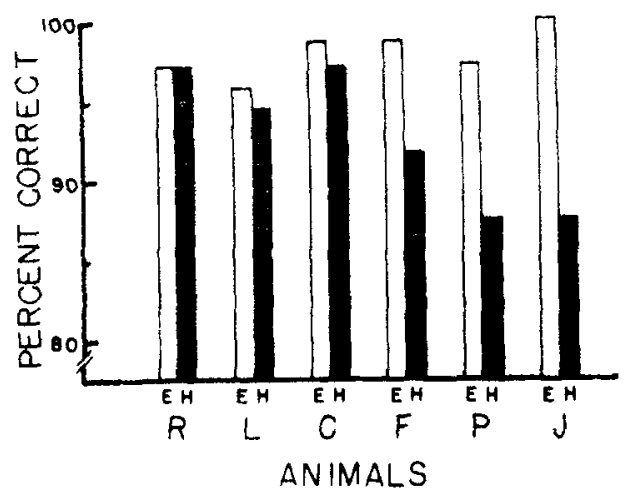

Figure 2. Performance for the six animals, Roscoe (R), Lucy (L), Coco (C), Fifi (F), Peewee (P), and Jocko (J), on the "easy" (E) and "hard" (H) sequences. The order of the animais was ananged arbitrarily from left to right as the difference in performance on the two conditions increased.

time ago. However, with a larger sample set, the probability is greater that the incorrect comparison stimulus did not appear as sample in the immediately preceding trials (assuming randomization of sample and comparison stimuli). In other words, large sample sets tend to generate "easy" sequences, while the converse is true for small sample sets.

Etkin and D'Amato (1969) reported no differences in DMTS performance when sample-set size was varied between two and four stimuli. However, because of their treatment order, practice effects could have masked any potential differences.

\section{Method}

The subjects, apparatus, and basic DMTS task were the same as described in Experiment $I$. The only differences were the lengths of the retention intervals, the number of stimuli used, and the fact that a 5-sec time-out signaled by dimming of the houselight was imposed between all trials.

Each session contained 12 2-sec delays and 12 long delays, all spent in darkness. The long delays were of $60 \mathrm{sec}$ duration for Lucy, Fifi, Coco, and Jocko, and 90 sec duration for Roscoe and Peewee. The main variable of interest was sample-set size, and within a given daily session, an animal was exposed to either two, three, or seven different stimuli used as samples and comparisons. A particular sample-set size was used for 2 consecutive days; thus, six daily sessions completed a block of data, with two blocks of 12 sessions in all. Drawn from a pool of seven stimuli (triangle, vertical line, horizontal line, square, circle, dot, and plus), different pairs of stimuli were used for each day of two-stimulus testing, and different triads were used for each day of three-stimulus testing. Pairs and triads were pseudo-randomly selected for each subject independently to minimize contribution of variance by any specific combinations of stimuli and their associated discriminability. The red and blue stimuli of the previous experiment were not included in the stimulus pool because of their high discriminability from the form stimuli. However, they were included along with the seven form stimuli in a maximum of 14 days pretraining.

\section{Results and Discussion}

Lucy and Coco were not included in the data analysis because of attentional deficits in observing the sample, thus invalidating their data as reflecting memory 


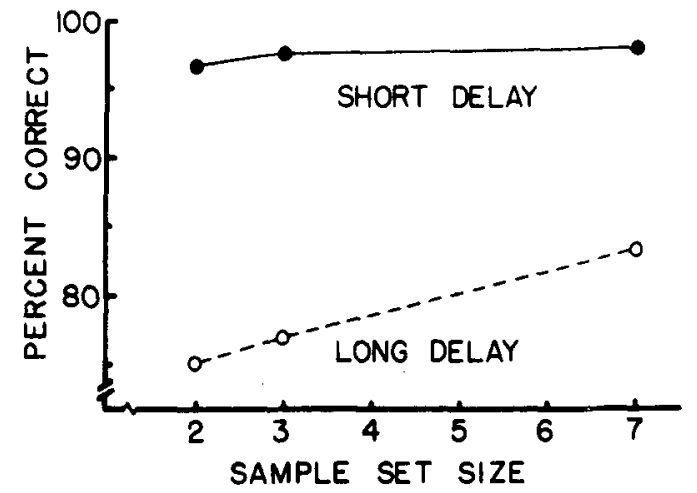

Figure 3. Matching performance as a function of sample-set size and delay length.

processes. Lucy developed the habit of throwing her head back while working the microswitch and pressing the sample key. Coco worked the microswitch from the right front corner of the chamber and pressed the sample key without looking at it. The decision to drop these subjects was made before the analysis was done.

An analysis of variance was performed on arc sine transformed data. This transformation was used because scores had an upper limit of 12 , which limited the range within which they could vary. The main effects were sample-set size, delay, blocks, and subjects. A trend analysis, corrected for unequal intervals (Gaito, 1964), was done on the sample-set size variable.

All main effects were significant: (1) the linear component of sample-set size, $\mathrm{F}(1,48)=5.357, \mathrm{p}<.05$; (2) delay, $\quad F(1,48)=141.157, \quad \mathrm{p}<.001 ;$ (3) blocks, $F(1,48)=4.387, \quad p<.05 ; \quad$ and (4) subjects, $F(3,48)=3.817, p<.05$. The only significant interaction of interest was Delay by Blocks, $F(1,48)=6.98$, $p<.05$. Performance on long delays was better in the second block than in the first block.

The results of the analysis showed that the linear component accounted for $99 \%$ of the sample-set size variance and that performance was indeed better in sessions having larger sample sets. The average percentage of correct responses for the 2-, 3-, and 7 -sample sets were $85.7,87.2$, and 90.6 , respectively. Figure 3 plots matching performance as a function of delay interval and sample-set size.

These results are interpreted to mean that small sample sets generate a greater amount of interference due to confusions in judging the temporal recency of stimuli. A small sample set makes the discrimination of most recent sample a harder one. For an animal working with a two-sample set, the same two stimuli always appeared at the end of the retention interval. Thus, there were no differential retrieval cues from trial to trial, and the task was reduced to a pure discrimination of recency. In sessions with larger sample sets, the probability is much reduced that a given trial's incorrect comparison stimulus occurred as sample in temporal contiguity with the current trial's sample, making confusion of sample recency less likely.

\section{GENERAL DISCUSSION}

The results of these experiments support the idea that temporal discrimination is an important process in DMTS and similar paradigms. Generally, monkeys perform better under conditions which facilitate their ability to form temporal discriminations.

The kind of retention evidenced by DMTS and related tasks is somewhat analogous to what Tulving (1972) has called "episodic memory." According to Tulving, episodic memory contains information about "temporally dated episodes or events, and temporal-spatial relations among these events." In a DMTS trial, at the time of the retention test, the task confronting the animal is to decide which of two stimuli has most recently served in the role of sample. For longer retention intervals, in a multiple-trial session, this decision becomes more difficult because the sample recency for the two stimuli becomes more similar (i.e., the temporal-spatial relation between the two stimuli, as sample, becomes hard to discriminate).

Discrimination of the temporal ordering of events may also be an important process in human STM paradigms. Murdock (1961) found that intrusion errors in a Peterson and Peterson (1959) type task demonstrate a marked temporal gradient, with intrusions from immediately prior trigrams being most common. Presumably, immediately preceding trigrams are more easily confusable with the target trigram because of their close temporal proximity. Also, Loess and Waugh (1967) found decreasing proactive interference as the ITI increased, with proactive interference being essentially absent when 2 -min ITIs were used. Longer ITIs result in wider temporal spacing of target items, which would allow for better discrimination of their relative occurrence in time (cf. Jarrard \& Moise, 1971).

One advantage of the temporal discrimination hypothesis is that it would seem to provide a basis for theoretical continuity between STM and LTM. Gleitman (1971) has suggested that forgetting (of temporal recency) is the explanation for proactive interference, rather than vice versa. For example, if an animal learns one task, then learns a second task, and is tested for retention later, it is usually said that a tendency to perform the first task demonstrates a proactive interference effect for memory of the second task (cf. Spear, 1971). It may simply be the case that as the retention interval increases, it is easier for the animal to forget which task it has learned more recently.

Winograd (1971) was more explicit concerning the role of temporal discrimination in LTM. He pointed out that forgetting can be viewed as a failure of discrimination. Assuming that animals follow the strategy of "doing what was last done successfully," the problem then becomes discriminating what was done last from what was done previously. He also stated that it may be more fruitful to look at temporal parameters in relative rather than absolute terms. For example, where 
an animal learns two consecutive tasks and is tested at some retention interval after learning the second task, the important factor would be a "ratio of recency" between: (a) the time from Task 1 to the retention test, and (b) the time from Task 2 to the retention test. Possibly, the relativity of temporal events should be a more important consideration than absolute retention intervals.

If both STM and LTM can be shown to be under the control of discrimination processes of a temporal nature, then a powerful common denominator of the two types of memory will have been identified. Although it is unlikely that any single variable can account for the full range of memory phenomena, a careful assessment of the role of temporal discrimination in memory processes should prove both interesting and valuable.

\section{REFERENCES}

Atkinson, R. C., \& Wickens, T. D. Human memory and the concept of reinforcement. In $R$. Glaser (Ed.), The nature of reinforcement. New York: Academic Press, 1971.

Broadbent, D. E. Perception and communication. London: Pergamon Press, 1958.

Broadbent, D. E. Flow of information within the organism. Journal of Verbal Learning and Verbal Behavior, 1963,2, 34-39.

Broadbent, D. E. Decision and stress. London: Academic Press, 1971.

Brown, J. Some tests of the decay theory of immediate memory. Quarterly Journal of Experimental Psychology, 1958, 10, 12-21.

D'Amato, M. R. Delayed matching and short-term memory in monkeys. In G. H. Bower (Ed.), The psychology of learning and motivation: Advances in research and theory Vol. 7 . New York: Academic Press. 1973.

D'Amato, M. R., \& O'Neill, W. Matching behavior: Some methodological problems. Behavior Research Methods \& Instrumentation, $1970,2,162-164$.

D'Amato, M. R., \& Worsham, R. W. Delayed matching in the capuchin monkey with brief sample durations. Learning and Motivation, 1972, 3, 304-312.

Etkin, M. W., \& D'Amato, M. R. Delayed matching-to-sample and short-term memory in capuchin monkeys. Journal of Comparative and Physiological Psychology, 1969, 69, 544-549.
Gaito, J. Unequal intervals and unequal $n$ in trend analyses. Psychological Bulletin, 1964, 63, 125-127.

Gleitman, H, Forgetting of long-term memories in animals. In $\mathrm{W}$ K. Honig and P. H. R. James (Eds.), A nimal memory. New York: Academic Press, 1971.

Herman, Louis M., \& Gordon, Judith, A. Auditory delayed matching in the bottlenose dolphin. Journal of the Experimental Analysis of Behavior, 1974, 21, 19-26.

Jarrard, L. E., \& Moise, S. L. Short-term memory in the monkey. In L. E. Jarrard (Ed.), Cognitive processes of nonhuman primates. New York: Academic Press, 1971.

Loess, H., \& Waugh, N. C. Short-term memory and intertrial interval. Journal of Verbal Learning and Verbal Behavior, $1967,6,455-460$.

Massaro, D. W., \& Kahn, B. J. Affects of central processing on auditory recognition. Journal of Experimental Psychology, $1973,97,51-58$.

Murdock, B. B. Retention of individual items. Journal of Experimental Psychology, 1961, 62, 618-625.

Murdock, B. B. Short-term memory. In G. H. Bower (Ed.), The psychology of learning and motivation: Advances in research and theory. New York: Academic Press, 1972.

Peterson, L. R., \& Peterson, M. J. Short-term retention of individual verbal items. Journal of Experimental Psychology $1959,58,193-198$.

Salzberg, P. M., Parks, T. E., Kroll, N. E. A., \& Parkinson, S. R. Retroactive effects of phonemic similarity on short-term recal of visual and auditory stimuli. Journal of Experimental Psychology, 1971,91, 43-46.

Spear, N. E. Forgetting as retrieval failure. In W. K. Honig and P. H. R. James (Eds.), Animal memory. New York: Academic Press, 1971.

Tulving, E. Episodic and semantic memory. In E. Tulving \& W. Donaldson (Eds.), Organization of memory. New York: Academic Press, 1972.

Weiskrantz. L. A long-term view of short-term memory in psychology. In G. Horn and R. A. Hinde (Eds.), Short-term changes in neural activity and behavior. Cambridge: Cambridge University Press, 1970.

Wickelgren, W. A. Multi-trace strength theory. In D. A. Norman (Ed.), Models of human memory: New York: Academic Press, 1970 .

Winograd, E. Some issues relating animal memory to human memory. In W. K. Honig and P. H. R. James (Eds.), A nimal memory. New York: Academic Press, 1971.

Worsham, R. W., \& D'Amato, M. R. Ambient light, white-noise, and monkey vocalization as sources of interference in visual short-term memory of monkeys. Journal of Experimental Psychology, 1973, 99, 99-105.

(Received for publication June 13, 1974; accepted September $30,1974$. 